\title{
Silicon photonics transceivers with integrated hybrid lasers
}

\author{
J-M Fédéli1 ${ }^{1}$ L Virot ${ }^{1,2,3}$, G.H. Duan ${ }^{5}$, L Vivien² ${ }^{2}$ D. Thomson ${ }^{6}$, J-M Hartmann ${ }^{1}$, C.Jany ${ }^{5}$,
} P.Grosse ${ }^{1}$, A. Le Liepvre ${ }^{5}$, W.Bogaerts ${ }^{4}$, G.Reed ${ }^{6}$, D.Van Thourhout ${ }^{4}$, F.Lelarge ${ }^{5}$,

${ }^{1}$ CEA, LETI, Minatec Campus, 17 rue des Martyrs, F-38054 Grenoble, France ${ }^{2}$ Institut d'Electronique Fondamentale (IEF), Univ. Paris-Sud, CNRS, Bât 220, F-91405 Orsay France ${ }^{3}$ STMicroelectronics, Silicon Technology Development, Crolles, France

${ }^{4}$ Photonic Research Group,- Ghent University, Ghent, Belgium

${ }^{5}$ III-V Lab, a joint lab of Alcatel-Lucent Bell Labs France, Thales Research and CEA, Avenue A. Fresnel, 91767 Palaiseau, France ${ }^{6}$ ECS/ORC, University of Southampton, Southampton, Hampshire, UK

Phone: +33 438786879 E-mail: jean-marc.fedeli@cea.fr

\section{Introduction}

Submicron silicon photonics have generated an increasing interest in recent years, mainly for optical telecommunications or for optical interconnects in microelectronic circuits. The rationale of silicon photonics is the reduction of the cost and energy of communications systems through the integration of photonic components and an electronic integrated circuit (IC) on a common chip (telecommunications applications), or the enhancement of IC performances with the introduction of optics inside a high performance chip (core to core communications), or low cost sensors. By co-integrating optics and electronics on the same chip, high- functionality, high-performance and highly integrated devices can be fabricated with a well-mastered microelectronics fabrication process. The FP7 HELIOS project aims to combine a photonic layer with a CMOS circuit by using microelectronics fabrication processes. A first goal was to develop high performance generic building blocks for a broad range of applications: WDM sources by III-V/Si heterogeneous integration [1], fast modulators [2,3] and detectors [4], passive circuits and packaging. With these building blocks, a transmitter with an $\mathrm{InP}$ on $\mathrm{Si}$ laser and a 16 channel receiver have been assembled.

\section{Receiver}

This receiver is imaged in Figure 1: A 2D surface grating couples the light coming from a single mode fiber SMF fiber into the circuit and separates the two polarizations while transforming the TM polarization into TE. Identical $200 \mathrm{GHz} 16$ channel AWGs receive the two input signals and demultiplex the guided TE modes. The two 16 output waveguides are then connected to 16 Ge photodiodes.

We have developed a self-aligned process for the fabrication of the waveguides using two photolithography steps with a $193 \mathrm{~nm}$ stepper and two Si dry etching steps for the fabrication of gratings and waveguides on optical SOI substrates from SOITEC (220nm Si on top of $2 \mu \mathrm{m}$ Buried OXide). Then cavities are defined for the selective epitaxial growth of Germanium. This is achieved by deposition of a silica layer which is etched at the end of waveguides. In order to achieve direct coupling, the silicon floor of the cavities is etched down to 50nm on top of the BOX. Germanium was then selectivity grown in the cavities and Chemical Mechanical Polishing used to adjust the thickness. A thick layer of silica was then deposited and windows opened in it down to the Ge layer underneath. In a self-aligned process, the doped regions ( $\mathrm{N}$ and $\mathrm{P}$ ) of the lateral PIN Ge photodetector were then defined by ion implantation of Phosphorus and Boron in the defined openings. The separation of the openings thus defines the width of the Ge intrinsic region. A $1 \mu \mathrm{m}$ thick $\mathrm{SiO} 2$ layer was then deposited and planarized before etching $400 \mathrm{~nm}$ diameter holes in it. These holes were filled with Ti/W and planarized in order to get $\mathrm{W}$ vias. A Ti/TiN/AlCu metal stack was deposited on this flat surface. DUV $248 \mathrm{~nm}$ lithography together with and $\mathrm{Cl} 2$ etching was used to fabricate the metallic pads.

The tests were performed on a $200 \mathrm{~mm}$ wafer prober. Using basic spirals, the propagation losses were statistically measured on the wafers. For $480 \mathrm{~nm} \times 220 \mathrm{~nm}$ clad- $^{-}$ ded waveguides, the losses were found at $2.3 \mathrm{~dB} / \mathrm{cm}$. The 2D grating coupler was adapted from the Gent University design [5] to the self-aligned technology (Figure 2). Using $2 \mathrm{x} 2 \mathrm{D}$ couplers mounted face to face, the Polarization Dependant Loss (PDL), the spectral response could be measured. The optimal efficiency for the $2 \mathrm{D}$ grating coupler was experimentally found to be $15 \%$ ( $~ 8 \mathrm{~dB}$ coupling losses) at $1550 \mathrm{~nm}$ with a $3 \mathrm{~dB}$ bandwidth of $55 \mathrm{~nm}$. The minimum PDL was measured at $\sim 1 \mathrm{~dB}$ at $\sim 1550 \mathrm{~nm}$. The 16 channels AWG with $200 \mathrm{GHz}$ separation is also adapted from a design from Gent University [5] with the technology and with the introduction of some absorbing sections. To reduce the crosstalk, an enlarged strip waveguide is used for the array part. So the crosstalk levels were measured at $-15 \mathrm{~dB}$, and the minimum center-channel insertion losses around $2.8 \mathrm{~dB}$.

The Ge photodiode is a butt coupled PIN lateral type of $10 \mu \mathrm{m}$ length. The photodiode sensitivity $\sim 0.8 \mathrm{~A} / \mathrm{W}$. $\mathrm{Ca}^{-}$ pacitance is in the $10 \mathrm{fF}$ range and dark current of the order of $20 \mathrm{nA}(-0.5 \mathrm{~V})$ as seen on figure 4 . The typical photodiode was connected to a $2 \mathrm{D}$ grating coupler and the polarization of the incoming was randomly changed. For this demonstrator, a separation of $1 \mu \mathrm{m}$ was selected and the measured bandwidth is around $20 \mathrm{GHz}$ which is comfortable for $10 \mathrm{~GB} / \mathrm{s}$ operation and should be also enough for $25 \mathrm{Gbit} / \mathrm{s}$ operation in new receivers.

The spectral characteristic of the receiver is shown in figure 2. With the losses and sensitivity of the basic blocks, the overall receiver sensitivity is in the order of $0.08 \mathrm{~A} / \mathrm{W}$ with a channel separation of $1.6 \mathrm{~nm}$, corresponding to 200GHz. The 2D grating coupler is responsible for the highest loss of sensitivity and should be optimized. Other solutions such as inverse taper or 1D grating coupler with separation of TE and TM coupled to a polarization rotator could be more efficient

\section{Transmitter}

An integrated tunable laser and MZM (ITLMZ) chip which consists of a single mode hybrid III-V/silicon laser [1], a silicon Mach-Zehnder (MZ) modulator and an optical output coupler have been designed and characterized (figure 3). The single-mode hybrid laser includes an InP waveguide providing light amplification, and a ring resonator allowing to achieve a single mode operation. Two Bragg reflectors etched 
on silicon waveguides form the laser cavity. The MZ modulator is based on $220 \mathrm{~nm}$ depletion type [2] and allows to modulate the output light emitted from the hybrid laser.

The fabrication process begins with $200 \mathrm{~mm}$ SOI wafers incorporating a $400 \mathrm{~nm}$ thick silicon waveguide layer on a $2 \mu \mathrm{m}$ BOX. DUV 193nm lithography and HBR etching of $180 \mathrm{~nm}$ silicon, allow the definition of rib waveguides for the coupling between the bonded III-V and silicon waveguides. By etching $120 \mathrm{~nm}$ silicon layer in the $220 \mathrm{~nm}$ defined level, rib waveguides for modulators are fabricated. The third step is the lithography and etching of $50 \mathrm{~nm}$ silicon layer, necessary for $220 \mathrm{~nm}$ stripe waveguides for Bragg gratings and output couplers. Then different ion implantation steps are carried out in order to make , p++, p, n and n++ doping for the modulators. An HDP oxide deposition on the wafers and a CMP are used to planarize the wafers. InP samples with the heterostructure are directly bonded to the planarized SOI wafer after the preparation of the surfaces. Then InP lasers are then processed, and metallization steps are performed for contacting the modulators, the heaters above ring resonators and the hybrid III-V/Si lasers. A NiCr material is deposited and etched above the ring resonator for the fabrication of heaters which thermally tune the resonance frequency of the ring resonator.

The laser threshold CW current is around $41 \mathrm{~mA}$ at $20^{\circ} \mathrm{C}$ and the output power coupled to the silicon waveguide is of around $1.8 \mathrm{~mW}$ for an injection current of $100 \mathrm{~mA}$. The maximum output power is around $3 \mathrm{~mW}$ at $20^{\circ} \mathrm{C}$, and the output power is higher than $0.5 \mathrm{~mW}$ at $60^{\circ} \mathrm{C}$. Single mode operation with SMSR larger than $35 \mathrm{~dB}$ is achieved

With the increase of current, the temperature of the silicon waveguide and the surrounding materials increase, leading to increased mode effective index and hence a shift of the resonance wavelength. As a result, the selected cavity mode will jump to another one with the lowest threshold. Figure 4 plots the lasing wavelength as a function of the heating power and a wavelength tuning range of $7 \mathrm{~nm}$ was achieved. The ITLMZ is tested by modulating one arm, at $10 \mathrm{~Gb} / \mathrm{s}$ using a peuso-random binary sequence (PRBS) of a length of $27-1$. The BER measurement is performed for 8 different wavelengths by changing the injection current into the heater.

The extinction ratio of all those wavelengths varies from 6 to $10 \mathrm{~dB}$. Figure 5 shows the BER curves for all the wavelengths and also a reference curve for a directly modulated laser, measured using a high sensitive receiver including an avalanche photodiode. One can see that several channels can achieve error free operation with BER $<10^{-9}$. The power level difference to achieve the same BER among all channels is around $4 \mathrm{~dB}$

\section{Acknowledgements}

The research leading to these results has received funding from European Community's under grant agreement $n^{\circ} 224312$ HELIOS.

\section{References}

[1] M. Lamponi \&al, "Low-threshold heterogeneously integrated InP/SOI laser with a double adiabatic taper coupler", IEEE Photonics Technology Letters, Vol: 24, P: 76 -78, 2012.

[2] D. J. Thomson, \&al, "High contrast 40Gbit/s optical modulation in silicon," Opt. Express 19, 11507-11516 (2011)

[3] M.Ziebell \&al, "40 Gbit/s low-loss silicon optical modulator based on a pipin diode", Optics Express Vol. 20, Issue 10, pp. 10591-10596 (2012)

[4] L.Vivien \& al, "Zero-bias 40Gbit/s germanium waveguide photodetector on silicon," Opt. Express 20, 1096-1101 (2012)

[5] W. Bogaerts \& al, "A polarization-diversity wavelength duplexer circuit in silicon-on-insulator photonic wires.," Optics express, vol. 15, no. 4, pp. 1567-78, Feb. 2007.

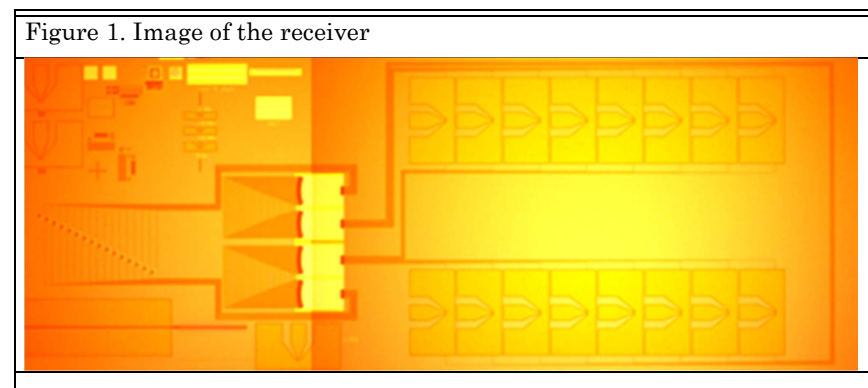

Figure 2. Photodiodes measurement after AWG.

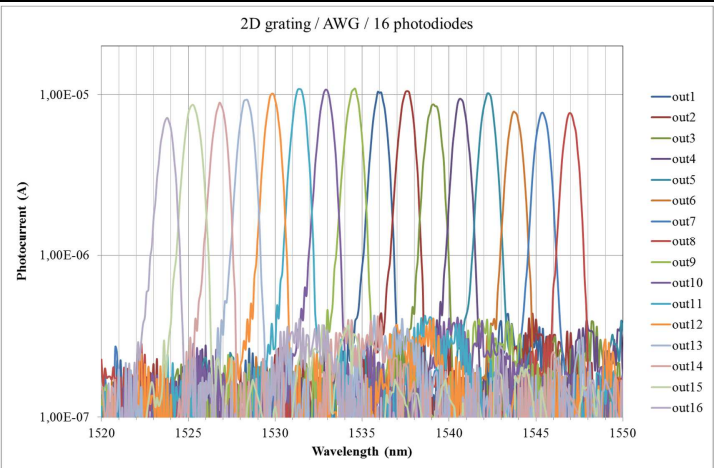

Figure 3. Image of the integrated tunable transmitter, incorporating a hybrid III-V/Si laser with wavelength tunability, and a silicon Mach-Zehnder modulator.

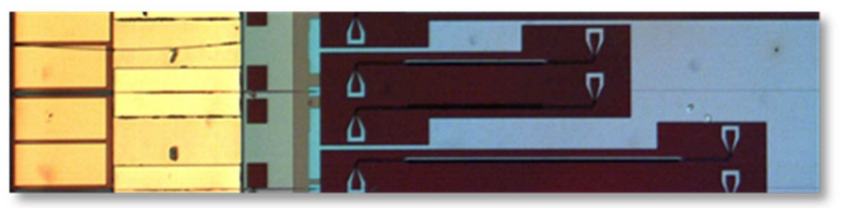

Figure 4. Lasing wavelength as a function of the heating power

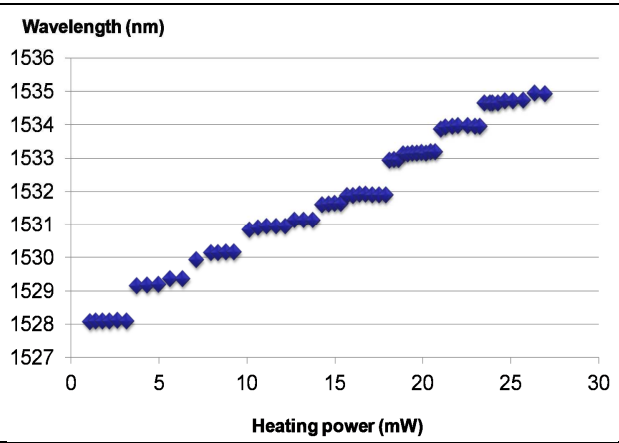

Figure 5. Bit error rate of the transmitter for different wavelength

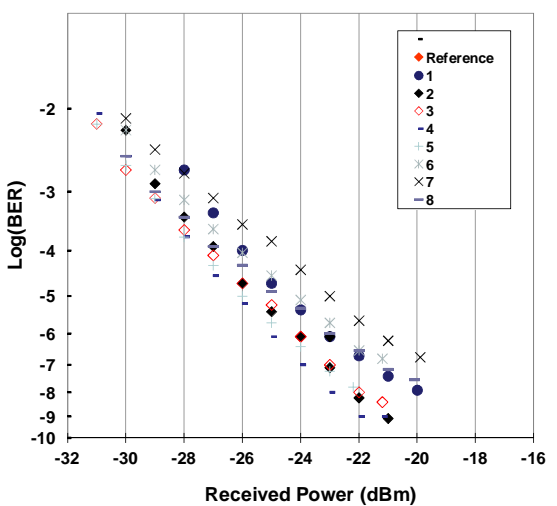

